\title{
Long-Term Survival of Participants in the Prostate Cancer Prevention Trial
}

\author{
Ian M. Thompson Jr, M.D, Phyllis J. Goodman, M.S, Catherine M. Tangen, Dr.P.H, Howard \\ L. Parnes, M.D, Lori M. Minasian, M.D, Paul A. Godley, M.D., Ph.D, M. Scott Lucia, M.D, and \\ Leslie G. Ford, M.D \\ Cancer Therapy and Research Center, University of Texas Health Science Center at San \\ Antonio, San Antonio (I.M.T.); Southwest Oncology Group Statistical Center and Fred Hutchinson \\ Cancer Research Center, Seattle (P.J.G., C.M.T.); Division of Cancer Prevention, National \\ Cancer Institute, Bethesda, MD (H.L.P., L.M.M., L.G.F.); Division of Hematology/Oncology, \\ University of North Carolina School of Medicine, School of Public Health, and the Lineberger \\ Comprehensive Cancer Center, Chapel Hill (P.A.G.); and the Department of Pathology, \\ University of Colorado, Denver School of Medicine, Denver (M.S.L.).
}

\begin{abstract}
BACKGROUND—In the Prostate Cancer Prevention Trial (PCPT), finasteride significantly reduced the risk of prostate cancer but was associated with an increased risk of high-grade disease. With up to 18 years of follow-up, we analyzed rates of survival among all study participants and among those with prostate cancer.
\end{abstract}

METHODS-We collected data on the incidence of prostate cancer among PCPT participants for an additional year after our first report was published in 2003 and searched the Social Security Death Index to assess survival status through October 31, 2011.

RESULTS-Among 18,880 eligible men who underwent randomization, prostate cancer was diagnosed in 989 of 9423 (10.5\%) in the finasteride group and 1412 of 9457 (14.9\%) in the placebo group (relative risk in the finasteride group, $0.70 ; 95 \%$ confidence interval [CI], 0.65 to $0.76 ; \mathrm{P}<0.001)$. Of the men who were evaluated, $333(3.5 \%)$ in the finasteride group and 286 $(3.0 \%)$ in the placebo group had high-grade cancer (Gleason score, 7 to 10) (relative risk, 1.17; $95 \% \mathrm{CI}, 1.00$ to $1.37 ; \mathrm{P}=0.05)$. Of the men who died, 2538 were in the finasteride group and 2496 were in the placebo group, for 15 -year survival rates of $78.0 \%$ and $78.2 \%$, respectively. The unadjusted hazard ratio for death in the finasteride group was $1.02(95 \% \mathrm{CI}, 0.97$ to $1.08 ; \mathrm{P}=$ 0.46). Ten-year survival rates were $83.0 \%$ in the finasteride group and $80.9 \%$ in the placebo group for men with low-grade prostate cancer and $73.0 \%$ and $73.6 \%$, respectively, for those with highgrade prostate cancer.

\footnotetext{
Copyright $@ 2013$ Massachusetts Medical Society. Address reprint requests to Dr. Thompson at the Cancer Therapy and Research Center, Suite U627, 7979 Wurzbach Rd., San Antonio, TX 78229, or at thompsoni@uthscsa.edu..

Disclosure forms provided by the authors are available with the full text of this article at NEJM.org.

No other potential conflict of interest relevant to this article was reported.
} 
CONCLUSIONS-Finasteride reduced the risk of prostate cancer by about one third. High-grade prostate cancer was more common in the finasteride group than in the placebo group, but after 18 years of follow-up, there was no significant between-group difference in the rates of overall survival or survival after the diagnosis of prostate cancer. (Funded by the National Cancer Institute.)

With the advent of prostate-specific antigen (PSA) testing in the late 1980s, the rate of diagnosis of prostate cancer rose dramatically. Currently, a man in the United States has a $16.5 \%$ lifetime risk of receiving a diagnosis of prostate cancer. ${ }^{1}$ The timing and magnitude of the $44 \%$ reduction in prostate-cancer mortality after the widespread adoption of PSA testing suggest that both screening and treatment improvements have contributed to this decline. ${ }^{2}$ Unfortunately, treatments for prostate cancer (radiation and surgery) are associated with a substantial risk of side effects, including sexual, urinary, and bowel complications, that can dramatically affect quality of life. ${ }^{3}$ Even active surveillance, the treatment of choice for many men with low-risk prostate cancer, represents a substantial health care burden, since it requires regular PSA testing, clinic visits, and periodic prostate biopsies, with a 2 to $4 \%$ risk of sepsis associated with each biopsy. ${ }^{4}$ Notably, the cost of active surveillance is not less than that of radical surgery or brachytherapy. ${ }^{5}$

Another approach that complements early-detection efforts is prostate-cancer prevention. With 241,740 prostate-cancer cases detected in the United States in 2012, a prevention strategy could have a meaningful public health effect. ${ }^{1}$ One method to reduce the risk of prostate cancer in the general population is the use of fin asteride (Proscar, Merck), a 5areductase inhibitor. In the Prostate Cancer Prevention Trial (PCPT), finasteride reduced the relative risk of prostate cancer by $24.8 \%$ (with 803 cancers in 4368 men evaluated in the finasteride group vs. 1147 cancers in 4692 men evaluated in the placebo group). ${ }^{6}$ Of concern was a relative increase of $26.9 \%$ in the risk of high-grade prostate cancers in the finasteride group, as compared with the placebo group (with high-grade prostate cancers developing in 280 of 4368 participants and 237 of 4692 participants, respectively). Subsequent analyses showed that the use of finasteride improved the sensitivity of PSA testing, prostate biopsy, and digital rectal examination for the detection of prostate cancer and improved the sensitivity of PSA testing and prostate biopsy for the detection of high-grade disease. ${ }^{7-9}$ This improved sensitivity may be the result of a reduction in PSA levels in men receiving finasteride who have benign enlargement and subsequent shrinkage of the prostate. Despite multiple analyses suggesting that detection bias accounted, at least in part, for the observed increase in the rate of high-grade tumors in the finasteride group, concern regarding this potential risk has all but eliminated the use of finasteride for prostate-cancer prevention. ${ }^{10-12}$

Given that 18 years had elapsed since the first PCPT participant underwent randomization, we undertook an analysis of survival in the two study groups to seek any evidence of an increased risk of death among men in the finasteride group, since such an increase would be a potentially accurate indicator of an increase in the risk of high-grade (and hence more lethal) cancer. We sought to update the number of prostate cancers detected since the inception of the study through an additional year of follow-up after the primary report was published in 2003 and to analyze rates of both overall survival and survival after the diagnosis of prostate cancer during the trial. 


\section{METHODS}

\section{Follow-Up of Participants}

From January 1994 through May 1997, a total of 18,882 men underwent randomization in the PCPT. During the trial, men were followed semi-annually by means of study-site visits and telephone calls at each intervening 3-month and 9-month interval. The men underwent annual digital rectal examination and measurement of PSA. At the end of 7 years, all the men in whom prostate cancer had not been diagnosed were offered an end-of-study biopsy. Data collection and prostate-cancer assessments continued until June 2004, providing up to 10 years of continuous observation for the majority of the study participants.

In 2005, 2 years after the publication of the primary report, ${ }^{6}$ we initiated a long-term followup study ${ }^{13}$ involving men in whom prostate cancer had been diagnosed during the study, with the objectives of estimating the time to metastases and the difference in all-cause and prostate-cancer mortality among men in the finasteride group, as compared with those in the placebo group. The results of this follow-up study, which was approved by the institutional review board at each study center, are reported here.

All the study participants provided written informed consent.

At the time that the follow-up study was initiated, many study centers had closed, and the statistical center did not have direct contact with the participants. As a result, enrollment was lower than expected, and because of a lack of feasibility, the long-term follow-up study was closed on May 1, 2009, and all active data collection was stopped.

\section{DIAGNOSIS OF PROSTATE CANCER}

The primary end point of the PCPT was histo-logically confirmed prostate cancer, including prostate cancer that was detected during the course of the 7-year treatment period and prostate cancer that was detected by means of an endof-study prostate biopsy, as reported previously. ${ }^{6}$ Our current study includes all detected prostate cancers, including those that did not meet the definition of the primary end point, until data collection ended. The majority of prostate-biopsy specimens were centrally reviewed by independent expert genitourinary pathologists who were unaware of the study-group assignments. We graded prostate cancers using the Gleason score, with the score for each tumor determined by the study pathologist. A high-grade tumor was defined as one with a Gleason score of 7 to 10, and a low-grade tumor as one with a Gleason score of 2 to 6 .

\section{DEATHS}

During the PCPT, when the statistical center was informed of a participant's death, clinical research associates were asked to collect source documentation to abstract the cause of death. Source documents included a death certificate at a minimum but preferably a narrative summary that was reviewed by the end-point review committee, which then ascertained the cause of death. To enhance survival data, the institutional review board at the statistical center approved a search of the Social Security Death Index (SSDI), which was performed in May 2012 to assess survival status through October 31, 2011 (because of an 
approximate 6-month lag in data reporting). ${ }^{14}$ The search included all men participating in the PCPT for whom a Social Security number was available and who were known to be alive at the end of the study. Data that were submitted in cluded the participant's full name, birth date, and Social Security number.

\section{STUDY OVERSIGHT}

The PCPT was designed in 1992 by investigators at the National Cancer Institute, SWOG (formerly the Southwest Oncology Group), and other participating cooperative groups. SWOG investigators gathered statistical data with oversight from the second and third authors. This report is a post hoc analysis that was not prespecified in the original protocol and was designed by the first three authors. Merck provided finasteride and placebo for the original study but did not participate in the design or oversight of this study, in the analysis of the data, or in the decision to submit the manuscript for publication.

\section{STATISTICAL ANALYSIS}

We used a measure of relative risk to estimate the association between study group and a diagnosis of prostate cancer and high-grade prostate cancer. All the men in the two study groups were included in the at-risk analysis. The PCPT protocol-specified primary analysis of the comparison between prostate-cancer treatments required that only men in whom prostate cancer had been diagnosed within the 7-year study period or who had a negative end-of-study biopsy result be included in the comparison, as was reported in $2003 .^{6}$

However, in the current analysis, we evaluated some cancers that were detected outside of the original, prespecified analysis of data from the 7-year study period, and we no longer required an end-of-study biopsy. The most unbiased approach was to include in the analysis of the updated estimate of relative risk all eligible men who had undergone randomization.

For the survival analysis, survival was calculated from the date of randomization to the date of death from any cause. Data for all men who were last known to be alive or who had died after October 31, 2011, were censored on October 31, 2011, because of the lag in updating the SSDI and changes to the SSDI in November 2011 mandating that states were no longer permitted to share data on deaths with the Social Security Administration. ${ }^{15,16}$ Data for men without a Social Security number were censored on the last date they were known to be alive, according to PCPT follow-up data. In the survival analysis for men in whom prostate cancer was diagnosed, survival was calculated from the date of the initial diagnosis to the date of death from any cause or the date of data censoring. We could not calculate causespecific mortality since data on the majority of deaths came from the SSDI, which does not include cause of death.

A Cox model was used to estimate hazard ratios for death associated with the two treatments, after adjustment for risk factors. The covariates that were included in the model for overall survival were the age at study entry as a continuous variable, race (black vs. nonblack), and a time-dependent variable for the diagnosis of prostate cancer. (Race and ethnic group were self-reported.) The covariates that were included in the model for survival after a prostate-cancer diagnosis were the age at diagnosis as a continuous variable, race (black vs. nonblack), a family history of prostate cancer (yes vs. no), the Gleason score (2 to 
6 vs. 7 to 10), and a term for the interaction between treatment and Gleason score. All reported $\mathrm{P}$ values are two-sided, and a $\mathrm{P}$ value of less than 0.05 was considered to indicate statistical significance. All analyses were performed with the use of SAS software, version 9.2 (SAS Institute). The proportional-hazards assumption for both models was tested within the PROC PHREG module.

\section{RESULTS DIAGNOSIS OF PROSTATE CANCER}

Figure 1 shows the categorization of participants according to study group and disease status and identifies which participants were included in the current analysis, as well as in the primary PCPT report. ${ }^{6}$ From the time of the original report through the end of data collection in June 2004, an additional 187 cancers were diagnosed in the finasteride group and 265 in the placebo group; of these cancers, high-grade tumors were diagnosed in 47 men in the finasteride group and 39 in the placebo group.

Of 18,880 eligible men who underwent randomization in the original study, prostate cancer was diagnosed in 2401: 989 of 9423 (10.5\%) in the finasteride group and 1412 of 9457 (14.9\%) in the placebo group (relative risk in the finasteride group, $0.70 ; 95 \%$ confidence interval $[\mathrm{CI}], 0.65$ to $0.76 ; \mathrm{P}<0.001)$. Of these cancers, $333(3.5 \%)$ in the finasteride group and $286(3.0 \%)$ in the placebo group were assessed as high-grade (Gleason score, 7 to 10) (relative risk, $1.17 ; 95 \% \mathrm{CI}, 1.00$ to $1.37 ; \mathrm{P}=0.05$ ). Relative-risk estimates for a diagnosis of any prostate cancer or high-grade prostate cancer in the current analysis and the original report are presented in Table 1.

\section{DEATHS}

A total of 5034 deaths were reported: 2538 in the finasteride group and 2496 in the placebo group. At the time of the SSDI search, 16,526 men were recorded in the database as being alive, and 16,136 (97.6\%) had provided a Social Security number, a full name, or both. The 390 men (196 in the finasteride group and 194 in the placebo group) for whom neither a full name nor a Social Security number was available had been evaluated at one institution that prohibited the release of these data. A total of 3422 deaths (68.0\% of all deaths) were found during the SSDI search performed in May 2012.

At the time of randomization (1994 to 1997), the median age of participants was 63.2 years. As of October 31, 2011, among the men who were still alive, the median age was 78.7 years. The 15 -year survival rate was $78.0 \%$ in the finasteride group and $78.2 \%$ in the placebo group (Fig. 2). The unadjusted hazard ratio for death in the finasteride group was 1.02 (95\% $\mathrm{CI}, 0.97$ to $1.08 ; \mathrm{P}=0.46$ ); after adjustment for age, race, and a diagnosis of prostate cancer, the hazard ratio was 1.03 ( $95 \% \mathrm{CI}, 0.98$ to $1.09 ; \mathrm{P}=0.26$ ).

The median age at the time of prostate-cancer diagnosis was 70 years in the two study groups. Among men in whom prostate cancer had been diagnosed, the hazard ratio for death in the finasteride group was $1.01(95 \% \mathrm{CI}, 0.85$ to $1.20 ; \mathrm{P}=0.90)$; after adjustment for cancer grade, age at diagnosis, race, and a family history of prostate cancer, the hazard ratio was $0.93(95 \% \mathrm{CI}, 0.78$ to $1.12 ; \mathrm{P}=0.45)$ (Table 2$)$. At 10 years, the rate of survival among 
patients with any grade of prostate cancer was $79.3 \%$ in the finasteride group and $79.5 \%$ in the placebo group. When men were classified according to cancer grade, the 10-year survival rates were $83.0 \%$ in the finasteride group and $80.9 \%$ in the placebo group among those with low-grade prostate cancer and $73.0 \%$ and $73.6 \%$, respectively, among those with high-grade prostate cancer (Fig. 3).

When the results were limited to the subgroup of men in whom prostate cancer was diagnosed at the time of the original report, ${ }^{6}$ the results were unchanged. The test for interaction between treatment and cancer grade was not significant $(P=0.32)$, indicating that the between-group difference in the risk of death from high-grade disease was not significant. The rate of prostate-cancer-specific survival could not be calculated because of the small number of men for whom the cause of death was ascertained.

\section{DISCUSSION}

Although early detection of prostate cancer by means of PSA testing may lead to reduced mortality from the disease, it also leads to a substantial overdetection of cancer, most notably the diagnosis of low-grade tumors. Despite the evidence from one randomized clinical trial showing a significant reduction in the risk of death from prostate cancer with PSA testing, the risk of overdetection contributed greatly to the recommendation of the U.S. Preventive Services Task Force against PSA testing. ${ }^{17,18}$

It is also the risk of these low-grade tumors that appears to be significantly reduced with the use of finasteride. In the original PCPT, there was a relative reduction of 38\% in the risk of prostate cancer with a Gleason score of 2 to 6 (Table 1). A major lingering concern, despite the evidence of enhanced cancer detection in men receiving finasteride, was the observed absolute increase in the number of high-grade cancers that were diagnosed. ${ }^{19}$ In 2011, the Food and Drug Administration mandated revisions to the labels for 5a-reductase inhibitors stating that the drugs may increase the risk of high-grade prostate cancer and are not approved for the prevention of prostate cancer. ${ }^{12}$ Specifically, there was concern that the high-grade cancers detected among men receiving finasteride would be clinically more aggressive and thus more lethal. ${ }^{20}$ If the increase in high-grade prostate cancers was not a finasteride-driven artifact of detection but rather reflected new high-grade cancers induced by finasteride, some increase in mortality among men receiving finasteride should become obvious during long-term follow-up. ${ }^{21}$

With 1 additional year of disease ascertainment, our analysis continues to show a significant reduction in the risk of prostate cancer in the finasteride group as compared with the placebo group (10.5\% vs. $14.9 \%)$. At the same time, although the number of high-grade cancers was higher in the finasteride group than in the placebo group (333 vs. 286), the relative increase of $17 \%$ in the rate of high-grade cancers in the finasteride group in the follow-up period was lower than the rate of $27 \%$ in the primary study (although with different denominators for the number of participants, the rates are not directly comparable). With up to 18 years of follow-up from the inception of the PCPT, we found no significant increase in the risk of death among men receiving finasteride (hazard ratio, 1.02; $\mathrm{P}=0.46$ ). The 15 -year rate of survival in each of the two groups was approximately $78 \%$. 
Among men in whom prostate cancer was diagnosed, there was no significant betweengroup difference in the rate of death after the date of diagnosis (hazard ratio with finasteride, $1.01 ; \mathrm{P}=0.90$ ). The hazard ratio for death among men with high-grade cancer in the placebo group, as compared with the finasteride group, was 0.94 (95\% CI, 0.70 to $1.27 ; \mathrm{P}=0.68$ ). The 10-year estimate of the rate of survival was approximately $79 \%$ in each of the two study groups. The diagnosis of a high-grade tumor in the screened population had a good prognosis, and there was no obvious difference in survival between the two groups. However, the wide confidence interval for the hazard ratio means that we cannot rule out a $30 \%$ decreased risk to a $27 \%$ increased risk of death in the placebo group, as compared with the finasteride group.

This study and its analysis have limitations. The source for the majority of the reported deaths was the SSDI. Because of the change in the SSDI in November 2011, missing Social Security numbers for 390 men (196 in the finasteride group and 194 in the placebo group), and the possibility of errors in the data entry of Social Security numbers, some of the deaths may not have been identified. However, there is no reason to believe that deaths would be missing differentially according to study group. The cause of death for the majority of men was not available, and as a result prostate-cancer-specific mortality cannot be determined. Since the total number of high-grade cancers was small and there were only a total of 177 deaths in this subgroup, there was not enough information to formally test noninferiority.

Data from 18 years of follow-up showed that the use of finasteride over a period of 7 years in a general population of men with a median age at study entry of 63.2 years reduced the risk of prostate cancer but did not significantly affect mortality. This reduction in risk was due entirely to a relative reduction of $43 \%$ in the risk of low-grade cancer among men receiving finasteride, as compared with placebo. Although the prevention of these tumors did not appear to reduce overall mortality, increased diagnosis of low-grade prostate cancer is a problematic byproduct of PSA testing, in that treatment adds little, if any, benefit and in that all forms of therapy cause considerable burden to the patient and to society.

\section{Acknowledgments}

Supported by a Public Health Service grant (CA37429) from the Division of Cancer Prevention, National Cancer Institute.

Dr. Thompson reports receiving consulting fees from Ferring and being a named inventor on patent applications filed by his institution for a penile-prosthesis design for the management of erectile dysfunction and for a lab-on-achip procedure for point-of-service identification of biomarkers for prostate-cancer detection; and Dr. Tangen, receiving consulting fees from Amgen and Eli Lilly.

\section{References}

1. Siegel R, Naishadham D, Jemal A. Cancer statistics, 2012. CA Cancer J Clin. 2012; 62:10-29. [PubMed: 22237781]

2. Etzioni R, Gulati R, Tsodikov A, et al. The prostate cancer conundrum revisited: treatment changes and prostate cancer mortality declines. Cancer. 2012; 118:5955-63. [PubMed: 22605665]

3. Sanda MG, Dunn RL, Michalski J, et al. Quality of life and satisfaction with outcome among prostate-cancer survivors. N Engl J Med. 2008; 358:1250-61. [PubMed: 18354103]

4. Manecksha RP, Nason GJ, Cullen IM, et al. Prospective study of antibiotic prophylaxis for prostate biopsy involving >1100 men. Scientific World Journal. 2012; 2012:650858. [PubMed: 22645441] 
5. Ollendorf, DA.; Hayes, J.; McMahon, P.; Kuba, M.; Pearson, SD. Management options for low-risk prostate cancer: a report on comparative effectiveness and value. Institute for Clinical and Economic Review; Boston: Dec. 2009 (http://www.icer-review.org/index.php/Reports/lrpc.html)

6. Thompson IM, Goodman PJ, Tangen CM, et al. The influence of finasteride on the development of prostate cancer. N Engl J Med. 2003; 349:215-24. [PubMed: 12824459]

7. Thompson IM, Chi C, Ankerst DP, et al. Effect of finasteride on the sensitivity of PSA for detecting prostate cancer. J Natl Cancer Inst. 2006; 98:1128-33. [PubMed: 16912265]

8. Thompson IM, Tangen CM, Goodman PJ, et al. Finasteride improves the sensitivity of digital rectal examination for pros tate cancer detection. J Urol. 2007; 177:1749-52. [PubMed: 17437804]

9. Lucia MS, Epstein JI, Goodman PJ, et al. Finasteride and high-grade prostate cancer in the Prostate Cancer Prevention Trial. J Natl Cancer Inst. 2007; 99:1375-83. [PubMed: 17848673]

10. Redman MW, Tangen CM, Goodman PJ, Lucia MS, Coltman CA Jr, Thompson IM. Finasteride does not increase the risk of high-grade prostate cancer: a bias-adjusted modeling approach. Cancer Prev Res (Phila). 2008; 1:174-81. [PubMed: 19138953]

11. Hamilton RJ, Kahwati LC, Kinsinger LS. Knowledge and use of finasteride for the prevention of prostate cancer. Cancer Epidemiol Biomarkers Prev. 2010; 19:2164-71. [PubMed: 20699373]

12. FDA drug safety communication: 5-alpha reductase inhibitors (5-ARIs) may increase the risk of a more serious form of prostate cancer. 2011. (http://www.fda.gov/Drugs/DrugSafety/ ucm258314.htm)

13. ClinicalTrials.gov. Long-term follow-up of patients who were diagnosed with prostate cancer on PCPT. (http://clinicaltrials.gov/ct2/show/NCT00288106)

14. Quinn J, Kramer N, McDermott D. Validation of the Social Security Death Index (SSDI): an important readily-available outcomes database for researchers. West J Emerg Med. 2008; 9:6-8. [PubMed: 19561695]

15. Sack, K. Researchers wring hands as U.S. clamps down on death record access. Vol. 8. New York Times; Oct. p. 2012http://www.nytimes.com/2012/10/09/us/social-security-death-record-limitshinder-researchers.html?hp\&_r=0

16. Winn, D. Could the recent change in the availability of the Death Master File affect your research? Cancer Epidemiology Matters Blog. National Cancer Institute; http://blog-epi.grants.cancer.gov/ 2012/05/24/could-the-recent-change-in-availability-of-death-master-file-data-affect-your -research

17. Schröder FH, Hugosson J, Roobol MJ, et al. Prostate-cancer mortality at 11 years of follow-up. N Engl J Med. 2012; 366:981-90. [PubMed: 22417251] [Erratum, N Engl J Med 2012;366:2137.]

18. Moyer VA. Screening for prostate cancer: U.S. Preventive Services Task Force recommendation statement. Ann Intern Med. 2012; 157:120-34. [PubMed: 22801674]

19. Kramer BS, Hagerty KL, Justman S, et al. Use of 5-alpha-reductase inhibitors for prostate cancer chemoprevention: American Society of Clinical Oncology/ American Urological Association 2008 clinical practice guideline. J Clin Oncol. 2009; 27:1502-16. [PubMed: 19252137] [Erratum, J Clin Oncol 2009;27:2742.]

20. Theoret MR, Ning Y-M, Zhang JJ, Justice R, Keegan P, Pazdur R. The risks and benefits of 5areductase inhibitors for prostate-cancer prevention. N Engl J Med. 2011; 365:97-9. [PubMed: 21675880]

21. Cuzick J. Long-term follow-up in cancer prevention trials (It ain't over 'til it's over). Cancer Prev Res (Phila). 2010; 3:689-91. [PubMed: 20522799] 


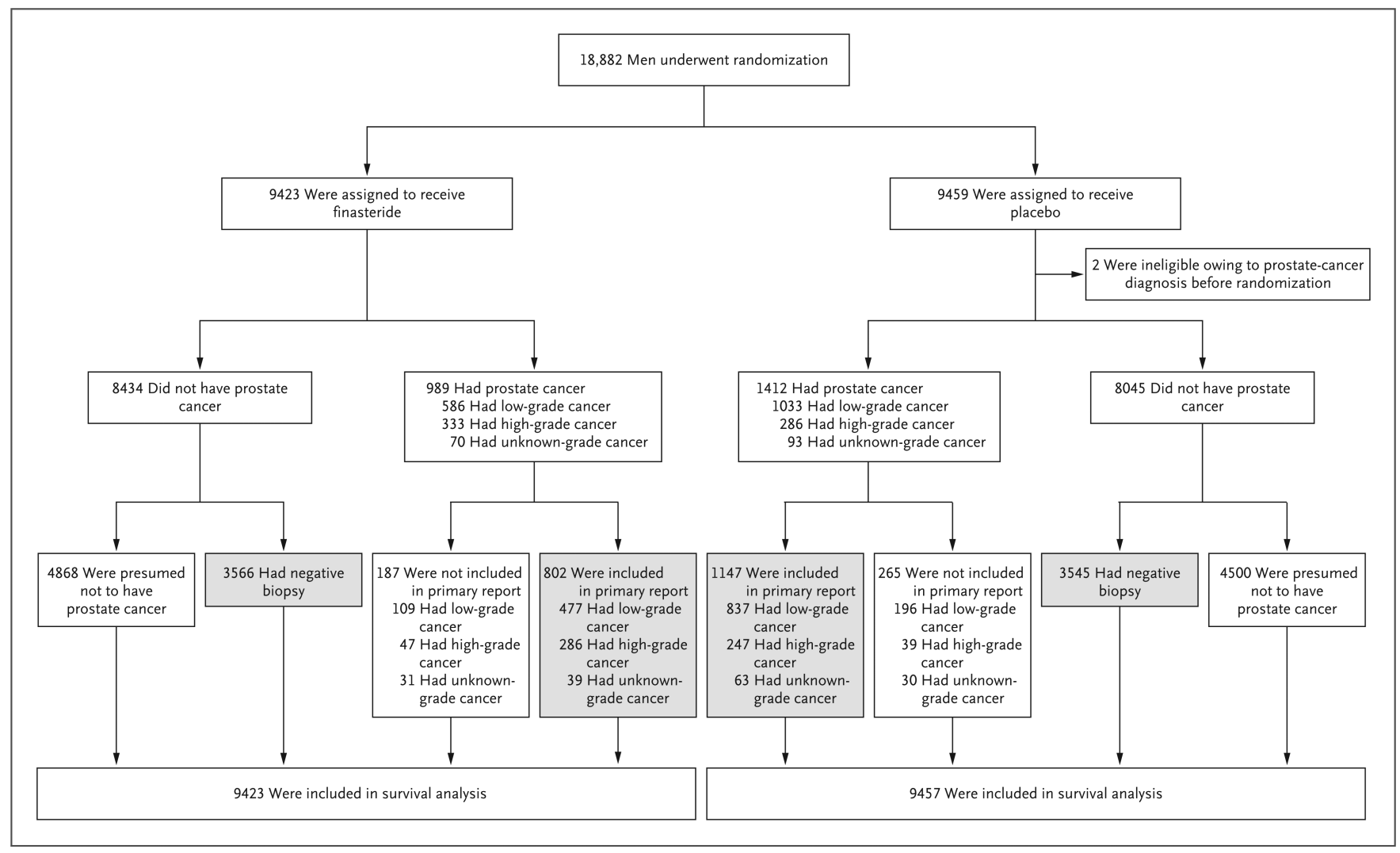

Figure 1. Enrollment and Outcomes

Data in the boxes with shading were included in the primary 2003 report of the Prostate Cancer Prevention Trial (PCPT). All other results are from the current follow-up study. Men with a negative biopsy result underwent the procedure before March 2003, the month in which data were censored for the primary report. Men who were presumed not to have prostate cancer did not undergo a biopsy at the end of the study. One participant in the finasteride group who was classified as having low-grade prostate cancer in the primary report was subsequently reclassified as having high-grade prostatic intraepithelial neoplasia. Among men who were included in the primary report, some prostate-cancer grades were changed after further central pathological review; the values presented here are the final confirmed values. Cancers that did not undergo central pathological review were classified as having an unknown grade. A total of 390 men (196 in the finasteride group and 194 in the placebo group) were evaluated at a single study center that prohibited the release of the study participant's full name and Social Security number. Data for these men were censored on the last date they were known to be alive on the basis of PCPT follow-up data. 


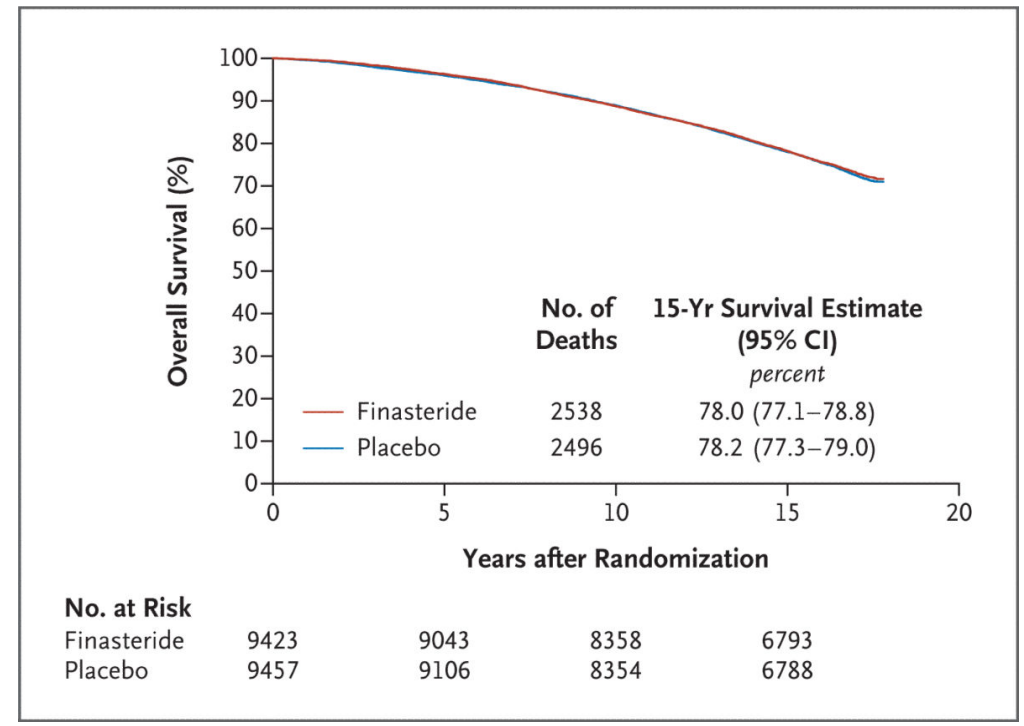

Figure 2.

Kaplan-Meier Curves for Overall Survival. 


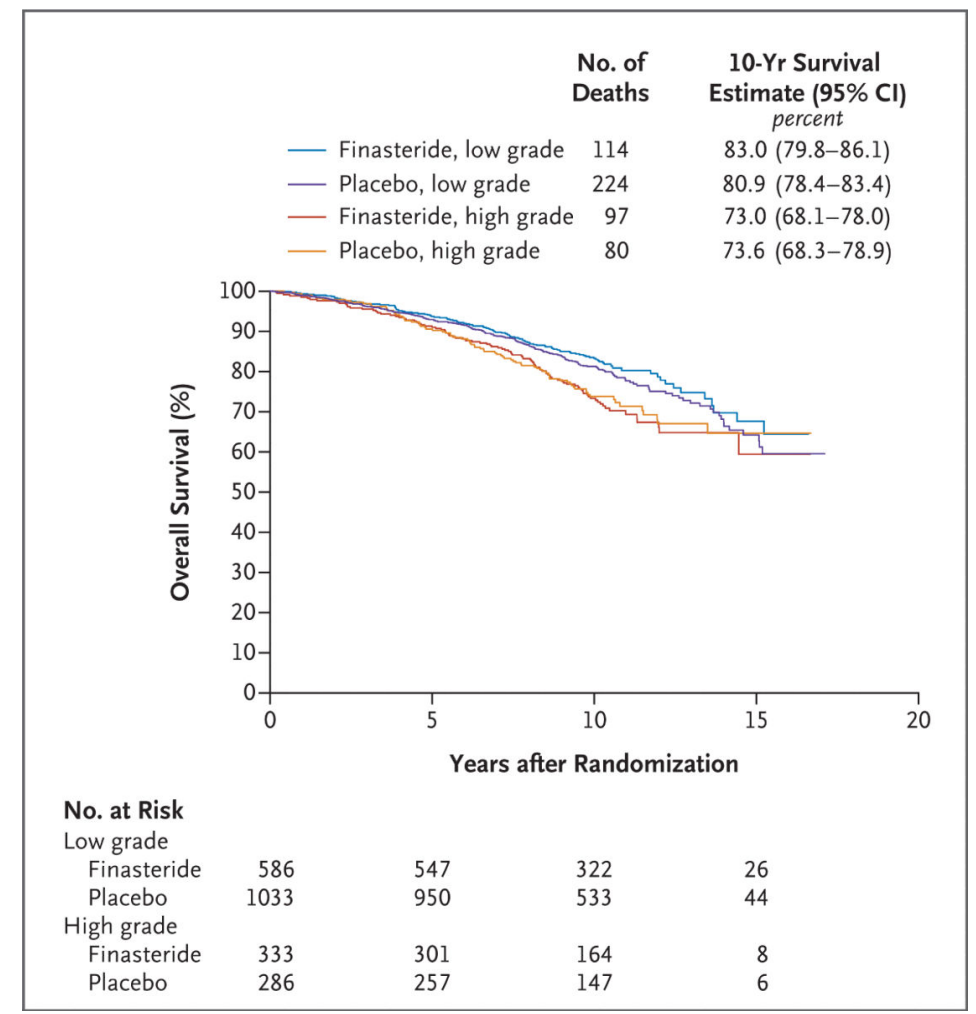

Figure 3. Overall Survival of Men with Prostate Cancer, According to Cancer Grade Low-grade cancers had a Gleason score of 2 to 6; high-grade cancers had a Gleason score of 7 to 10 . A total of 163 cancers with an unknown grade were excluded from this analysis.

Estimates of survival were reported at 10 years (rather than 15 years, as in Fig. 2) because of the small number of men who were evaluated at 15 years. 


\section{Table 1}

Relative Risk of Prostate Cancer in the Finasteride Group, as Compared with the Placebo Group, According to Cancer Grade.

\begin{tabular}{|lcccc|}
\hline Prostate-Cancer Grade & \multicolumn{2}{c}{ Primary 2003 Report } & \multicolumn{2}{c|}{ Current Study $^{\dagger}$} \\
& Relative Risk (95\% CI) & P Value & Relative Risk (95\% CI) & P Value \\
Any grade & $0.75(0.69-0.81)$ & $<0.001$ & $0.70(0.65-0.76)$ & $<0.001$ \\
Low grade & $0.62(0.56-0.68)$ & $<0.001$ & $0.57(0.52-0.63)$ & $<0.001$ \\
High grade & $1.27(1.07-1.50)$ & 0.005 & $1.17(1.00-1.37)$ & 0.05 \\
\hline
\end{tabular}

* Low-grade cancers had a Gleason score of 2 to 6; high-grade cancers had a Gleason score of 7 to 10.

${ }^{\dagger}$ Included in this analysis were men who had undergone end-point assessment, according to the protocol-specified window of inclusion. When all men who had undergone randomization were included, the relative risk in the finasteride group was 0.70 for any grade of prostate cancer $(\mathrm{P}<0.001), 0.58$ for low-grade cancer $(\mathrm{P}<0.001)$, and 1.19 for high-grade cancer $(\mathrm{P}=0.05)$.

\# Included in this analysis were eligible men who had undergone randomization and all prostate cancers detected during the follow-up period that extended through June 2004. 
Table 2

Hazard Ratios for Death from Any Cause and Death after Diagnosis of Prostate Cancer. ${ }^{*}$

\begin{tabular}{|c|c|c|c|}
\hline Variable & No. of Men Evaluated & Hazard Ratio $(95 \%$ CI) & P Value \\
\hline Death from any cause & 18,880 & & \\
\hline \multicolumn{4}{|l|}{ Finasteride vs. placebo } \\
\hline Unadjusted & & $1.02(0.97-1.08)$ & 0.46 \\
\hline Adjusted $^{\dagger}$ & & $1.03(0.98-1.09)$ & 0.26 \\
\hline Age at randomization & & $1.12(1.11-1.13)$ & $<0.001$ \\
\hline Black vs. nonblack race $\S$ & & $1.48(1.29-1.70)$ & $<0.001$ \\
\hline Diagnosis of prostate cancer ${ }^{I}$ & & $0.95(0.86-1.02)$ & 0.22 \\
\hline \multicolumn{4}{|l|}{ Death after diagnosis of prostate cancer } \\
\hline \multicolumn{4}{|l|}{ Finasteride vs. placebo } \\
\hline Unadjusted & 2,401 & $1.01(0.85-1.20)$ & 0.90 \\
\hline Adjusted $/ / * *$ & 2,238 & $0.93(0.78-1.12)$ & 0.45 \\
\hline High-grade vs. low-grade cancer $/ / \dagger \dagger$ & 2,238 & & \\
\hline High-grade cancer in finasteride group & & 1.00 (reference) & \\
\hline Low-grade cancer in finasteride group & & $0.64(0.49-0.84)$ & 0.001 \\
\hline High-grade cancer in placebo group & & $0.94(0.70-1.27)$ & 0.68 \\
\hline Low-grade cancer in placebo group & & $0.73(0.58-0.93)$ & 0.01 \\
\hline Age at diagnosis & & $1.11(1.09-1.12)$ & $<0.001$ \\
\hline Black vs. nonblack race & & $1.00(0.65-1.54)$ & 0.99 \\
\hline Family history of prostate cancer & & $0.91(0.73-1.14)$ & 0.42 \\
\hline
\end{tabular}

* Hazard ratios are for the finasteride group as compared with the placebo group, unless otherwise indicated. A hazard ratio of less than 1 indicates a reduced risk of death.

${ }^{\dagger}$ The hazard ratio was adjusted for age, race, and diagnosis of prostate cancer (time-dependent covariate).

the age at randomization was a continuous variable.

$\S_{\text {Race was self-reported. }}$

${ }^{I}$ Diagnosis of prostate cancer was added as a time-dependent covariate to account for the timing of the diagnosis.

"Men with an unknown grade of prostate cancer were excluded from the analysis.

$* *$

The hazard ratio was adjusted for cancer grade, age, race, and a family history of prostate cancer.

${ }^{\dagger \dagger}$ The hazard ratio was adjusted for age, race, and a family history of prostate cancer. 